\title{
L-INDEX: A Conceptual Index of Textual Data on MapReduce Platform
}

\author{
Dunlu Peng ${ }^{1}$, Kai Duan ${ }^{1,2}$ and Lei Xie ${ }^{1}$ \\ ${ }^{1}$ School of Optical-Electrical and Computer Engineering, \\ University of Shanghai for Science and Technology, Shanghai, 200093, China \\ ${ }^{2}$ The First Research Institute of Telecommunications Technology, \\ Shanghai, 200000, China \\ dlpeng@usst.edu.cn,duankai@yeah.net,leixie2007@126.com
}

\begin{abstract}
Although in past few decades, lots of research has been done on searching documents in textual data and there have been many commercial systems for retrieving data on the Web. Fast retrieving desired documents from massive textual corpora is still challenging. Indexing is the most commonly used way to improve the performance of retrieving; particularly an effective index structure can help retrieving data more quickly and effectively. This work proposes a novel index structure, named as L-INDEX, to conceptually index massive set of textual data. L-INDEX employs Form Concept Analysis to discover the relationships between documents and expresses the discovered relationships with lattice. To make our approach more applicable to process massive set of data, the structure is implemented in MapReduce environment which can fulfill computing tasks in an efficient distributed way. A set of algorithms is developed for creating, maintaining, storing L-INDEX and searching documents through it as well. A serial of experiments is conducted to verify the performance of our approach by comparing it with some other popular existing indexing structures. The experimental results demonstrate that the proposed approach can index massive set of textual data with an effective structure which supports querying tasks in an efficient way.
\end{abstract}

Keywords: Indexing structure, Textual data, Formal Concept Analysis, Lattice, MapReduce, Information Retrieval

\section{Introduction}

Document retrieval has gained lots of research attention for in past few decades, lots of research has been done on retrieving documents in textual corpora. To make retrieving efficiently, indexing is the most commonly used way to improve the performance of retrieving; particularly an effective indexing structure can help retrieving data more quickly and effectively. Building an effective indexing structure becomes a critical issue for guaranteeing retrieving data in a way with high quality and efficiency.

As the volume of data increases dramatically in some scenarios, such as on the Web, efficiently retrieving desired documents poses a giant challenge. However, indexing centrally could not ensure the performance of retrieving data in massive set, i.e., data in terabyte-scale, petabyte-scale or even more. To solve this kind of problems, people often resort to distributed indexing structures which index data on different machines and support retrieving in parallel $[4,5]$. Unfortunately, these distributing indexing techniques need fully machine local data which is difficult to achieve when a large number of machines are involved.

In recent years, some new distributing computing paradigms come into being, such as MapReduce [2] and its implementation Hadoop [3] which has been very popular in recent 
years, to gain higher retrieving performance. MapReduce is designed for distributing computing run over large datasets. It hides the complex details of parallel processing, such as fault tolerance and node availability, in its conceptual framework [6], which allows implementation of distributed computing much easier on top of it. Roughly, MapReduce processes computing tasks in two phases. In the first phase, it uses function Map to assign the computing task onto different machines over different subsets of data. MapReduce gains good throughput by using Distributed File System (DFS) to store data, because in DFS data is replicated in blocks across different machines, which makes the mapped processing, be accomplished as local as possible. Intermediate outcomes are also saved locally on the processing machine and are merged by a function called Reducer which is the job of the second phase. This mechanism makes implementations in MapReduce cheaper to develop and maintain than traditional parallel frameworks.

Building distributed indexing structure under MapReduce brings additional efficiency for document retrieval. Researchers have paid much attention to establishing distributed indexing approaches to indexing on MapReduce platform since Jeffery and Sanjay envisaged two possible interpretation of indexing in their original MapReduce paper [2]. For instance, Lin et al employed one of them for inverted indexing in their Ivory IR system [8]. Cafarella and Cutting deployed a MapReduce indexing strategy into their Nutch platform [7], using the Hadoop MapReduce implementation. In Terrier IR platform [9], McCreadie, et al., proposed a single-pass MapReduce indexing strategy which is similar to the single-pass indexing corpus scanning phase. These strategies provide efficient ways for searching in MapReduce in most cases. However, they lack considering the underlying relationships among data objects, which make improvement on the retrieving quality and efficiency.

As is shown in the literature [10, 11, 22], Formal Concept Analysis (FCA) is often applied to uncover the relationships between objects and the attribute. In this work, our goal is to develop distributed structure to efficiently support retrieving desired documents over massive dataset. We first discuss using FCA to discover the relationships among the data, and then employ Formal Concept Lattice (FCL) to connect the documents semantically with each other. Based on this, we build a distributed lattice-based indexing structure, called $L-I N D E X$, to conceptually index the data on MapReduce platform. Furthermore, we provide the algorithms for building, storing, maintaining L-INDEXand searching documents through $L-I N D E X$. We also study the competitor system to $L-I N D E X$, Lucene, and provide a performance comparison that shows $L$-INDEX to be superior.

The rest of this paper is organized as follows: in Section 2, we describe the state-of-the-art for distributed indexing textual data. Section 3 describes the definitions and fundamental theory for conceptually indexing textual data. Section 4 provides the algorithms of generating concepts from massive set of documents on MapReduce platform. We address issues on establishing, storing, maintaining L-INDEX and retrieving on it in Section 5. Section 6 presents the experiments conducted to testify the performance of $L-I N D E X$. Finally, we conclude our work and the future work in Section 7.

\section{Related Work}

This section lists some of the main structures for indexing documents in a large-scale corpus to improve the retrieving performance. We briefly mention the traditional structures involved in indexing processing (in Section 2.1), some general distributed structures (in Section 2.2) as well as the evolutions in distributed structures dedicated to indexing documents on MapReduce platform(in Section 2.3). We also address how our index structure, $L-I N D E X$, differs from them. 


\subsection{Traditional Index Structures}

To make retrieval of documents from repository efficiently, people often refer the original documents with suitable data structures which are collectively called an index. The most known structures employed to build the index of large-scale collection are inverted index [12] and signature index [19]. According to [20], compared with signature index, inverted index can evaluate queries more efficiently both in time and space. Therefore, in our work, we focus on inverted index that commonly used to index documents in IR systems.

Inverted index is nearly at the center of all today's full-text search engines and gives a term providing access to the list of documents that contain the term. For each term in its basic form, an inverted index consists of a posting list, which is associated the term with the documents in the collection. A posting list is comprised of individual postings, each of which consists of integer document-IDs (doc-IDs) and some useful statistic information. For Boolean retrieval, besides the document-IDs, no additional information is needed in the posting. Usually, the most common statistic information includes the term frequency or the time of the term occurrences in the document and the position of each occurrence of the term in the document. More complicated information comprises the field information (such as if the term occurred in the page title or not, which enables document ranking based on notions of importance during retrieval), or even the results of additional linguistic processing (for example, indicating that the term is part of a place name, to support address searches). In Web search engines, anchor text information (text associated with hyperlinks) is often appeared in the index as well [14]. Evidently, the size of an inverted index depends on the information each posting stores. If only frequency of terms is stored, the index size will be only a tenth of the size of original documents in the corpus, and nevertheless, the index size can easily be much larger than the original documents if the positional information of terms is stored in the index. Therefore, a lot of researches focus on developing effective techniques to compress data in inverted index [16].

When accomplishing a query during retrieval, the system searches the index for the posting lists which contain query terms and traverses the postings to calculate the result set. Boolean retrieval is the simplest case, in which only set operations (for example, union for boolean OR and intersection for boolean (AND) are needed to execute on postings lists. This process can be accomplished very efficiently because the postings are often sorted with doc-IDs. However, for a general query, query-document matching scores must be computed and parts of scores are stored in structures called accumulators. After all postings are processed, the system will return the top $k$ documents in a ranked list to the user. An obvious disadvantage of retrieving on inverted index is that too many postings might be examined during the query, which makes the retrieval with lower performance. Fortunately, to reduce the number of postings must be examined during the retrieval, many optimization strategies have been proposed for query evaluation (both approximate and exact) on inverted index in the literature $[15,16]$.

\subsection{General Distributed Index Structures}

The traditional inverted index depicted above is designed to run on single machine which generally has limited available memory. In some scenarios, such as in the context of Web, the document repository is too large to construct index efficiently on a single machine. It is practical for today's search engines to construct reasonable size of index using large computer clusters. The process of the construction is to use distributed indexing algorithms to partition the index across machines in the clusters. The strategies which the distributed indexing algorithms employ to implement the partition is either term-based or document-based [17].

A term-based index is also known as a global organized index, in which the dictionary of terms is partitioned into subsets and each subset resides at a node. The postings for the 
terms are kept at the node. This strategy allows a stream of queries with different query terms to be executed on a set of machines, which could support greater concurrency for the system. However, the greater concurrency can be easily surpassed by the cost of sending long posting lists between sets of nodes for merging when the system tries to accomplish multi-terms queries. There are lots of factors which determine load balancing for the partition, including frequencies of relative terms, the distribution of query terms and their co-occurrences. Most of these factors are hard to quantify, which makes indexing dynamically with the strategy more difficult.

Compared with term-based indexing, document-based indexing is more common implementation to partition, which makes each node contain a subset of documents in the repository. When a query comes, the system distributes it to all the nodes in the cluster, and then presents the results to the user after merging them from various nodes. It is clear this strategy trades more disk seeks for less inter-node communication. This approach could crack the weakness of term-based indexing. Unfortunately, there is a difficulty when using this approach to compute the global statistics for scoring, such as idf. The computation must be conducted across the entire corpus even though the index at any single node only contains a subset of the documents. Generally, in order to obtain the refresh information, the computation is distributed to background processes periodically at the nodes where the indexes are refreshed.

\subsection{Indexing in MapReduce}

Since Jeffery and Sanjay envisaged two possible interpretations of indexing documents in the original MapReduce paper [2], researchers have developed multiple structures based on the interpretations for indexing documents under MapReduce [7-9].

As described in [2], MapReduce employs two functions map and reduce to perform indexing documents. The main idea is described as follows: the map function parses each document and emits a sequence of <word, doc-ID>pairs, and then the reduce function accepts all pairs for a given word, sorts the corresponding doc-IDs and emits a <word, list (doc-ID) > pair, and finally the set of all output pairs forms a simple inverted index. However, the original paper does not claim how to store the statistic information, such as term frequencies, which makes the index more useful. According to the main idea of MapReduce, [21] discussed that indexing documents in MapReduce can be implemented in two ways: per-token indexing and per-term indexing.

Per-token Indexing From the perspective of per-token indexing, the map function would output a group of <term, doc-ID>pairs for each token in a document, and the reduce function returns the term frequencies after aggregating the doc-IDs where the term occurs. This strategy has the advantage of a very simple map phase, as it emits on a per-token basis, which is why it is referred as the per-token indexing strategy.

Suppose a term appears $t f$ times in a single document, then the <term, doc-ID>pair will be emitted $t f$ times. This makes the intermediate map data extremely large and increases the map-to-reduce network traffic, as well as spending more time aggregating.

Per-term Indexing Differs from the per-token indexing strategy, per-term indexing strategy emits once per unique term per-document with $\langle$ term, (doc-ID, $t f)>$ tuples, where tf is the term frequency of the term in the document. This significantly reduces the number of emit operations which have to be done. In addition, the reduce task for per-term indexes is also much simpler than for per-token indexes, as it only has to sort instances by document to obtain the final posting list sorted by ascending doc-ID.

Compared with per-token indexing strategy, it is apparently per-term indexing strategy improves the performance of retrieving single term. However, if we try to retrieve the documents that contain more than one specified terms, the system might have to send the request to all the nodes in the cluster, and then access the postings stored on each node. These operations are still very time-consuming without building an efficient structure to 
store the postings beforehand. Few off-the-shelf indexing strategies for MapReduce discuss how to improve the efficiency by taking advantages of the underlying relationships among documents. In this work, we propose a novel index structure, named as $L-I N D E X$, to conceptually index massive set of textual data. $L-I N D E X$ employs FCA to discover the relationships among textual documents, and expresses the discovered underlying relationships among documents with concept lattice. To make $L$-INDEX more applicable for managing massive corpora, the structure is implemented in MapReduce. Different from the existing indexing strategies in MapReduce, L-INDEX keeps the relationships among documents both at a node and the entire cluster. With this approach, both the network traffic and the time taken to search on a single node are reduced significantly, that improves the performance of the retrieval. In next section, we fist describe the sate-of-the-art the use of FCA (Formal Concept Analysis) in information retrieval, and then we introduce the fundamental theory of FCA we exploit in our work.

\section{FCA and Problem Description}

\subsection{Introducing FCA}

FCA (Formal Concept Analysis), whose foundation is the theory of concept lattice, is a mathematic method used to conceptually analyze data [1]. It utilizes formal concepts to model a domain with its intension and extension. Generally, the extension is formed by the objects, while the intension is composed of the attributes characterizing the objects. FCA is often used to uncover the implicit relationships among objects with concept lattice. So far, FCA has been successfully applied to a wide range of applications [22, 11]. Here, we describe how to use the fundamental theory of FCA to express the relationship among documents.

\subsection{Basic Theory}

Building the formal concept context, which is formed with objects, attributes and their relationships, is the first step to analyze data with FCA. We regard the documents in the corpus as the objects, the terms contained in at least one document as the attributes, and the appearance of a term in a document as the relationship. Therefore, the form concept context for document retrieval which we call it as document context can be defined as

Definition 1 The document context of a textual corpus is a triple $C(D, T, R)$, where $D$ refers to all the documents in the corpus, $T$ is the set of terms which appear in at least one document in $D$, and $R$ represents the relationships between the elements of $D$ and those of $T$, that is, $R=D \times T$. Let $d \in D, t \in T$ and $r \in R$ be the value of their relationship between $t$ and $c$. If $t$ appears in $d, r=1$, otherwise, $r=0$.

Example 1: Let $D=\{d o c 1, d o c 2, d o c 3, d o c 4, d o c 5\}$ be a set of documents (the corpus) and $T=\{$ printer, book, price, dictionary, car $\}$ be the set of terms that appear in the documents. The document context describes whether a term is contained in a document. Table 1 presents the document context of $D$. The names of the column are the terms in $T$, and the names of the rows are the doc-IDs in $D$. If the value of a cell equals 1 means that the term (column name) appears in the corresponding document of its row, otherwise, the term does not appear in the document.

Document context expresses the relationships between documents and terms well, however, it cannot depict the connections among the documents intuitively. In FCA, a concept lattice is often exploited to organize the objects according to the form context. Here, we employ it to connect the documents according to the document context. Figure 1 is the corresponding concept lattice of Table 1 . In this work, we try to index documents in a corpus by using concept lattice. To differ it from the ordinary concept lattices, this lattice-like index is called as L-INDEX. L-INDEX is formally depicted as follows.

Definition 2 Given a document context $C(D, T, R), L$ is the $L$-INDEX for indexing the 
documents in $D$ w.r.t $C(D, T, R)$, and has the following two properties:

- Property 1: Let $N\langle T, D\rangle$ be a node of $L$, where $T$ and $D$ are the intention and extension of $N$ respectively. $T$ and $E$ satisfy $f(T)=D$ and $g(D)=T$, where $N\langle T, D\rangle$ is called a formal concept or concept in $C(D, T, R)$.

- Property 2: Given any two adjacent nodes $N_{1}\left\langle T_{1}, D_{1}\right\rangle$ and $\left.N_{2}<T_{2}, D_{2}\right\rangle$ in $L$, if $T_{1}$ $\subset T_{2}$ and $D_{2} \subset D_{1}$ exist, $N_{1}$ is defined as a sub-index (or child) of $N_{2}$ or $N_{2}$ is a super-index (or parent) of $N_{1}$, denoted as $N_{1} \prec N_{2}$.

$$
\left\{\begin{array}{l}
f(T)=\{d \in D \mid \forall t \in T,(d, t) \in R\} \\
g(D)=\{t \in T \mid \forall d \in D,(d, t) \in R\}
\end{array}\right.
$$

Table 1. An Example of Document Context

\begin{tabular}{|c|c|c|c|c|c|}
\hline$D \times T$ & printer & book & price & dictionary & car \\
\hline$d o c_{1}$ & 1 & 1 & 1 & 0 & 0 \\
\hline$d o c_{2}$ & 1 & 0 & 1 & 0 & 1 \\
\hline$d o c_{3}$ & 1 & 0 & 0 & 1 & 0 \\
\hline$d o c_{4}$ & 0 & 0 & 0 & 1 & 1 \\
\hline$d o c_{5}$ & 1 & 0 & 0 & 1 & 1 \\
\hline
\end{tabular}

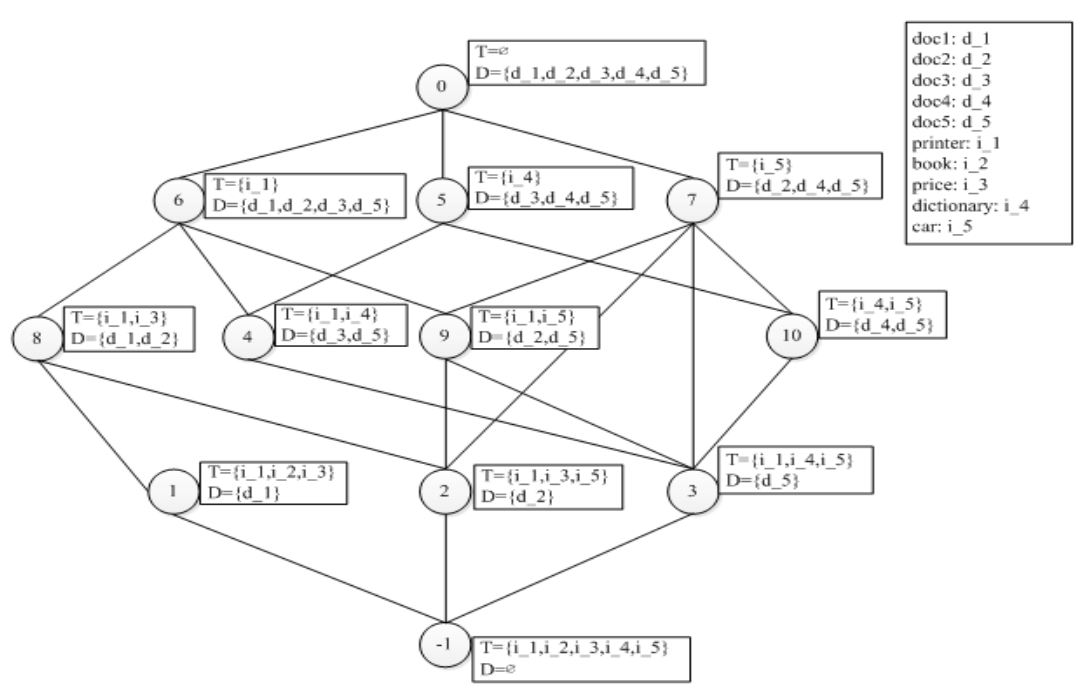

Figure 1. L-INDEX Corresponding to the Document Context Shown in Table 1

In $L$-INDEX, a node $N\langle T, D\rangle$ is corresponding to a formal concept in FCA. However, in order to keep identical with the term used in MapReduce, $T$ is also referred to as key of $N\langle T, D\rangle$ and $D$ is referred to as the value of $N\langle T, D\rangle$. Thus, in following sections, $N<$ key, value >will represent concepts alone unless otherwise stated.

Example 2: Let us take the two adjacent nodes, Node $2<$ (printer, price, car), $\left(d o c_{2}\right)>$ and Node $9<($ printer, car $),\left(d o c_{2}, d o c_{5}\right)>$ in Figure 1 for example. Obviously, the terms listed in the intension appear in the documents listed in the corresponding extension, that is, all the three terms of $\{$ printer, price, car $\}$ appear in $d o c_{2}$, and terms in $\{$ printer, car\} appear in both $d o c_{2}$ and $d o c_{5}$. Simultaneously, the key of Node 2 is \{printer, price, car $\}$ which contains that of Node $9,\{$ printer, car $\}$, while its value, $\left\{d o c_{2}\right\}$, is contained in that of Node $9,\left\{d o c_{2}, d o c_{5}\right\}$, therefore, Node $2<$ Node 9.

Now, we briefly describe how to retrieve documents containing a group of terms with $L-I N D E X$. For instance, when running "AND" query, if all the searched terms exist in the key of a node, the documents in its value are the expected ones to the users. The search starts from the top of the index, where the node has the largest extension and smallest intention, and traverses along the paths in which the key of nodes contain at least one 
common term with ones being searched. The traverse stops at the node whose key contains all the searched terms and returns its value, or stops at the bottom of the $L-I N D E X$ and returns null. Thus, we can see searching on the L-INDEX is very efficient, because it need not to traverse the entire index besides along the paths whose nodes' intensions have some common terms with the searched ones. This prunes the searching space significantly so that the cost of searchings' reduces evidently.

Example 3: In Figure 1, suppose we like to retrieve all the documents that contain the two terms printer and price, it starts at the top node named Node 0 and traverses along the path till it arrives at Node 8 whose key exactly equals $\{$ printer, price $\}$. Other nodes need not be traversed because their keys do not contain either of the two words being searched. Apparently, searching on L-INDEX is quite time-saving as most parts of index are pruned very early during the search.

\subsection{Problem Description}

The key step to index documents with an L-INDEX is to compute all the formal concepts or pairs of <key, value >for the nodes. Although searching on L-INDEX is very efficient, there still lacks effective approaches to computing the <key, value>pairs for large corpora. As far as we know, MapReduce provides high performance in large-scale computing [2] with large clusters. In this paper, we try to develop a set of effective distributed algorithms to efficiently compute the <key, value >pairs from a massive corpus in MapReduce. This research aims to build an L-INDEX for a massive corpus of documents by using MapReduce's advantage of high performance. Formally, we describe the problem with following description.

Given a massive corpus of textual documents $D$, the set of the terms appearing in the documents $A$ and a computer cluster with $n$ nodes. To build L-INDEX efficiently, we assign the task of its establishment onto different nodes in MapReduce. Formally, $D$ is partitioned into $n$ parts $D_{1}, D_{2}, \ldots, D_{n} \subset D$ and the corresponding term sets are $A_{1}, A_{2}, \ldots, A_{n}$ $\subset A$, each part is allocated into a different node of the cluster. Our goal is to design a map functions to build $L$-INDEX at each node and a reduce function to merge the sub-indexes gathering from different nodes in MapReduce platform. In addition, we also need to discuss how to fulfill search requests in the cluster and how to maintain the index when adding into or removing some documents from the corpus.

\section{Generating Formal Concepts in MapReduce}

To establish L-INDEX, formal concepts need be generated at first. As known to us, MapReduce provides an efficient distributed computing paradigm. In this section, we discuss how to generate formal concepts from massive document sets with MapReduce, which involves the tasks of effectively storing document context, designing a map and a reduce function special for generating formal concepts.

\subsection{Storage of Document Context}

After investigating the document context shown in Table 1, it is readily thinking of utilizing matrix to store document contexts. In some cases, however, especially when the corpus is immense, the matrix will be very sparse. It is because when there are massive documents in the corpus and each document only contains a small number of terms in the dictionary. In the worst situation, suppose there are $j$ documents on each node, $i$ terms each document, the size of each element of the matrix is $s$ bytes, and there is no common terms between any two documents. The number of terms in the matrix is $i \times j$, and the space needed to store the entire matrix is about $i \times j \times j \times s$ bytes, and only $\frac{i \times j \times s}{i \times j \times j \times s}=\frac{1}{j}$ of which is not vacant. For the low utilization of space, apparently, it is not suitable to store a massive document context with matrix directly. 
In this study, a document context is stored as a sequence of tuples $\left\{\left(r_{1}, c_{1}\right),\left(r_{1} c_{2}\right), \ldots,\left(r_{n}\right.\right.$, $\left.\left.c_{m}\right)\right\}$, where $\left(r_{i}, c_{j}\right)$ refers to document $d o c_{i}$ containing term $t_{j}$, (i.e., where the cell value is 1 in the table of document context). Obviously, this approach as against matrix is quite space-efficient. As an example, the document context shown in Table 1 is stored as $\{(1,1)$, $(1,2),(1,3),(2,1),(2,4),(3,1),(3,4),(4,4),(4,5),(5,1),(5,4),(5,5)\}$. This structure is very convenient to be processed for computing concepts from it. We will introduce the details in Section 4.2.

\subsection{Map Function: LMap}

One critical step of processing data using MapReduce is to develop an effective map function used to compute the pairs of $\langle k e y$, value $>$ at the nodes in the cluster. After examining the formal context (see Table 1) and the definition of L-INDEX (see Definition 2), we find that the computation boils down to extracting the common terms (i.e., keys) from the subsets of documents (i.e., values). However, for a given corpus, suppose it contains $n$ documents, there are at most $2 n$ combinations of the documents. It is clear that not all of the combinations make sense for generating formal concepts. Thus, if we use the brutal-force algorithms to generate the formal concepts without considering the facts, it is hard to accomplish the computation in acceptable time. A special algorithm is designed to generate the formal concepts.

In our method, we define a lexicographic order for all the subsets of the corpus. For conciseness, the corpus is simplified as $D=\{1,2, \ldots, n\}$ where each number in $D$ stands for a doc ID. Furthermore, elements in each subset of $D$ are also in lexicographic order. For example, suppose $A=\{1,4,6,9\}$ is a subset of $D, A$ cannot be written as $\{1,6,4,9\}$ or $\{1,4,9,6\}$. For any two subsets of the corpus, they are ordered in following way:

Definition 3 Suppose $A, B \subseteq D$, the elements in both $A$ and $B$ are in the lexicographic order, $x \in A$ and $y \in B$ are the first two elements which are not equal from the left side of $A$ and $B$. If $x>y$ exists, then $A$ is smaller than $B$ in the lexicographic order and denoted as $A<B \Leftrightarrow C(\exists i \in B-A, A \cap\{1,2, \ldots, i-1\}=B \cap\{1,2, \ldots, i-1\})$.

Example 4: Assume $A, B \subseteq D, D=\{1,2,3,4,5,6,7\}$ and $A=\{1,3,6,7\}, B=\{1,5,7\}$. As the first two unequal elements in $A$ and $B$ are 3 and 5, $B<A(i=3, A \cap\{1\}=B \cap\{1\}=$ $\{1\})$.

Clearly, Definition 3 defines a full order for the power set $2^{|D|}$, that is, if $A, B \subseteq D$ and $A$ $\neq B$, then $A<B$ or $A>B$ always holds.

To efficiently generate all the formal concepts, the first problem need be solved is to discover all the extensions with high efficiency. To accurately describe our algorithm, we introduce two expressions $A<_{i} B$ and $A \oplus i$ as follows:

Definition 4 Given $A, B \subseteq D, A<_{i} B$ is defined as $i \in B-A$ and $A \cap\{1,2, \ldots, \mathrm{i}-1\}=B \cap\{1$, $2, \ldots, \mathrm{i}-1\}$, and $A \oplus i$ is defined as $g(f((A \cap\{1,2, \ldots, i-1\}) \cup\{i\}))$.

According to the definition of $A<_{i} B$ and $A \oplus i$, the following assertions are derived:

$A 1$ : For any $i \in D, A<B \Leftrightarrow A<_{i} B$.

$A 2$ : If $A<_{i} B$ and $A<j C$, then $i<j \Rightarrow C<_{i} B$.

$A 3: i \notin A \Rightarrow A<A \oplus i$.

$A 4: A<_{i} B$ and $B$ is an extension $\Rightarrow A \oplus i \subseteq B$, then $A \oplus i \leq B$.

$A 5: A<_{i} B$ and $B$ is an extension $\Rightarrow A<_{i} A \oplus i$.

Since the above assertions have already been proved in the literature, we do not repeat it in this paper. For the readers who are interested in the proof, please refer to [1, 23]. We only apply the assertions to prove the theorem used in our algorithm. 

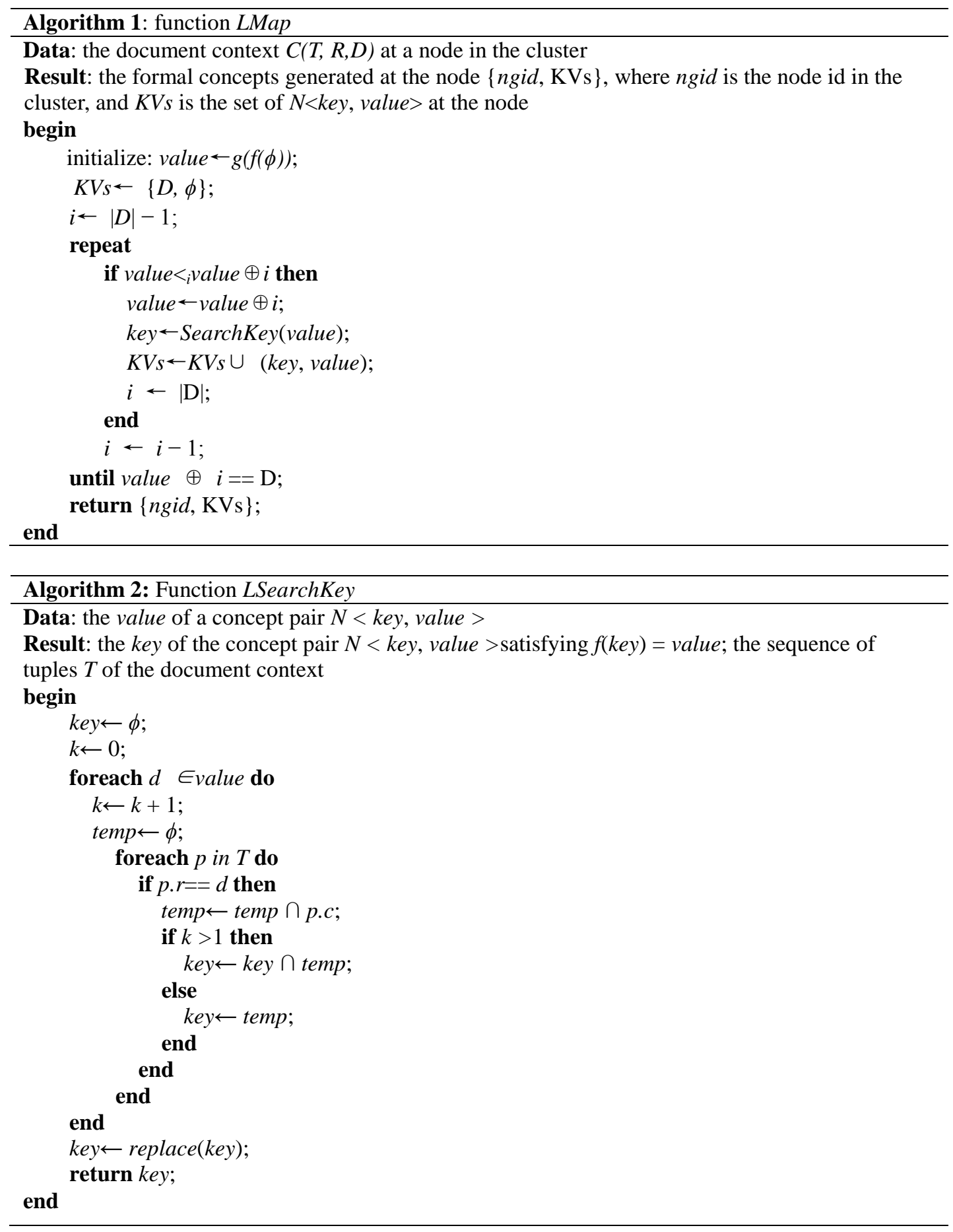

Theorem 1 The minimal extension, which is larger than a given subset $A \subset D$ in the lexicographic order, is $A \oplus i$, where $i$ is the greatest element satisfying $A<_{i} A \oplus i$.

Proof: Let $A^{+}$be the minimal extension larger than $A$ in the lexicographic order. There exists $i \in D$ which makes $A<_{i} A^{+}$hold. $A<_{i} A \oplus i$ for $A^{+}$is an extension (by $A_{5}$ ), and $A \oplus i$ $\leq A^{+}$(by $A_{4}$ ). According to Definition $4, A \oplus i$ must be an extension. Thus, with $A<A \oplus i$ $\leq A^{+}$and $A^{+}$is the minimal extension larger than $A$, we get $A \oplus i=A^{+}$. Now, let us prove $i$ is the maximal value, assume there is a $j \neq i$ that makes $A<_{j} A \oplus j$ exist. Therefore, $A \oplus j$ is also an extension larger than $A$. According to the minimality of $A^{+}$, we know $A^{+}<A \oplus j$, so that $A \oplus i<A \oplus j$ holds. Notice that $A<_{i} A \oplus i$ and $A<_{i} A \oplus j$ which leads $j<i$ (by $A_{2}$ ). 
On the basis of the assertions and Theorem 1, we present an algorithm for discovering the corresponding extension for a given subset $A \subseteq D$. The extension is the minimal extension which is larger than $A$ using the lexicographic ordering defined above. The algorithm starts with the minimal extension $g(f(\phi))$ of all the extensions followed by the discovery of the nearest subset that is larger than $g(f(\phi))$. No doubt that this subset must be another extension. Then, substitute this subset for the extension discovered in previous step, in the meantime, the subset is saved in the result extension set. Repeatedly do this substitution until the maximal extension $D$ itself is reached.

The detail of the map function, LMap, is described in Algorithm 1. After computing the extension of each concept, the algorithm invokes SearchKey to seek the common terms contained in all the documents in the extension. To make the return value to be more easily processed in MapReduce, instead of using the ids to stand for the terms in key, SearchKey transforms the ids to the terms themselves with function Replace (see Algorithm 2).

For the purpose of improving the performance of computing in reduce phase, the sets of concepts from different nodes are divided into various groups, and each set is labeled with the group ID, ngid, in the algorithm. After generating the formal concepts at all the nodes with LMap function, the computing shifts to the reduce phase. We discuss the concrete of reduce function, LReduce, in the next section.

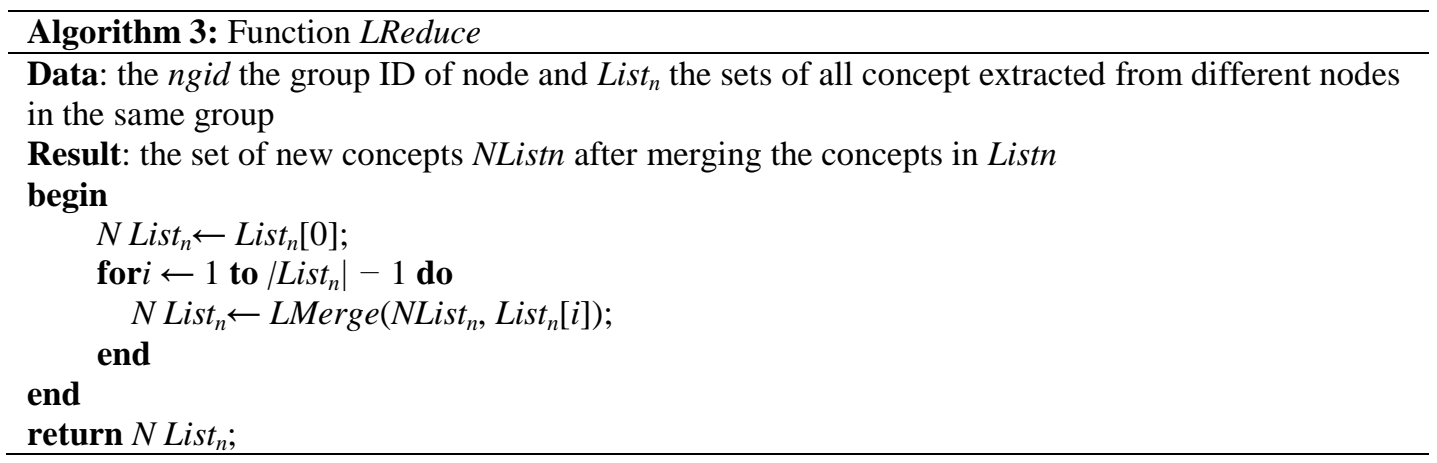

\subsection{Reduce Function: LReduce}

Map function fulfills computing formal concepts at a node, to reduce the network traffic, we need to merge the formal concepts originally from individual nodes to form larger concept sets. This task is performed in the reduce phase in MapReduce.

According to map function described in the preceding section, we know that the returned value from each node is in the form of $\{$ ngid, $K V s\}$, and $K V s$ is a set of concept pairs $\langle k e y, v a l u e>$. Suppose during the reduce phase we try to merge the concepts from the nodes labeled with same ngid. Given two concepts $N_{1}\left\langle k e y_{1}, v_{a l u e_{1}}\right\rangle, N_{2}\left\langle k e y_{2}\right.$, $v_{\text {value }}>$ from different two nodes in the same group. It is visible to find that the new concepts would be $N_{\text {merge }(1,2)}<k e y_{1} \cap k e y_{2}$, value ${ }_{1} \cup$ value $_{2}>$ or $N_{\text {merge }(1,2)}<k e y_{1} \cup$ key $_{2}$, value $_{1} \cap$ value $_{2}>$. To reduce the workload of entire cluster, we ensure that each document takes part in the extraction of concepts on a single node in the cluster, this makes value $\cap$ value $_{2}=\phi$. Therefore, actually, it only generates $N_{\text {merge(1,2) }}<k e y_{1} \cap k e y_{2}$, value $_{1} \cup$ value $_{2}>$ as the new concepts. Taking this observation, we develop an algorithm to merge concepts in the reduce function, LReduce. The function is depicted in Algorithm 3, and its main idea is described as follows: Suppose $\left\{n g i d, K V s_{1}\right\}$ and $\left\{n g i d, K V s_{2}\right\}$ are two sets of concepts returned from two different nodes in the same group, and $N_{1}<$ key $_{1}$, value $_{1}>\in K V s_{1}, N_{2}<$ $k e y_{2}$, value ${ }_{2}>\in K V s_{2}$, when merging $N_{1}$ and $N_{2}$, the following rules are kept: 


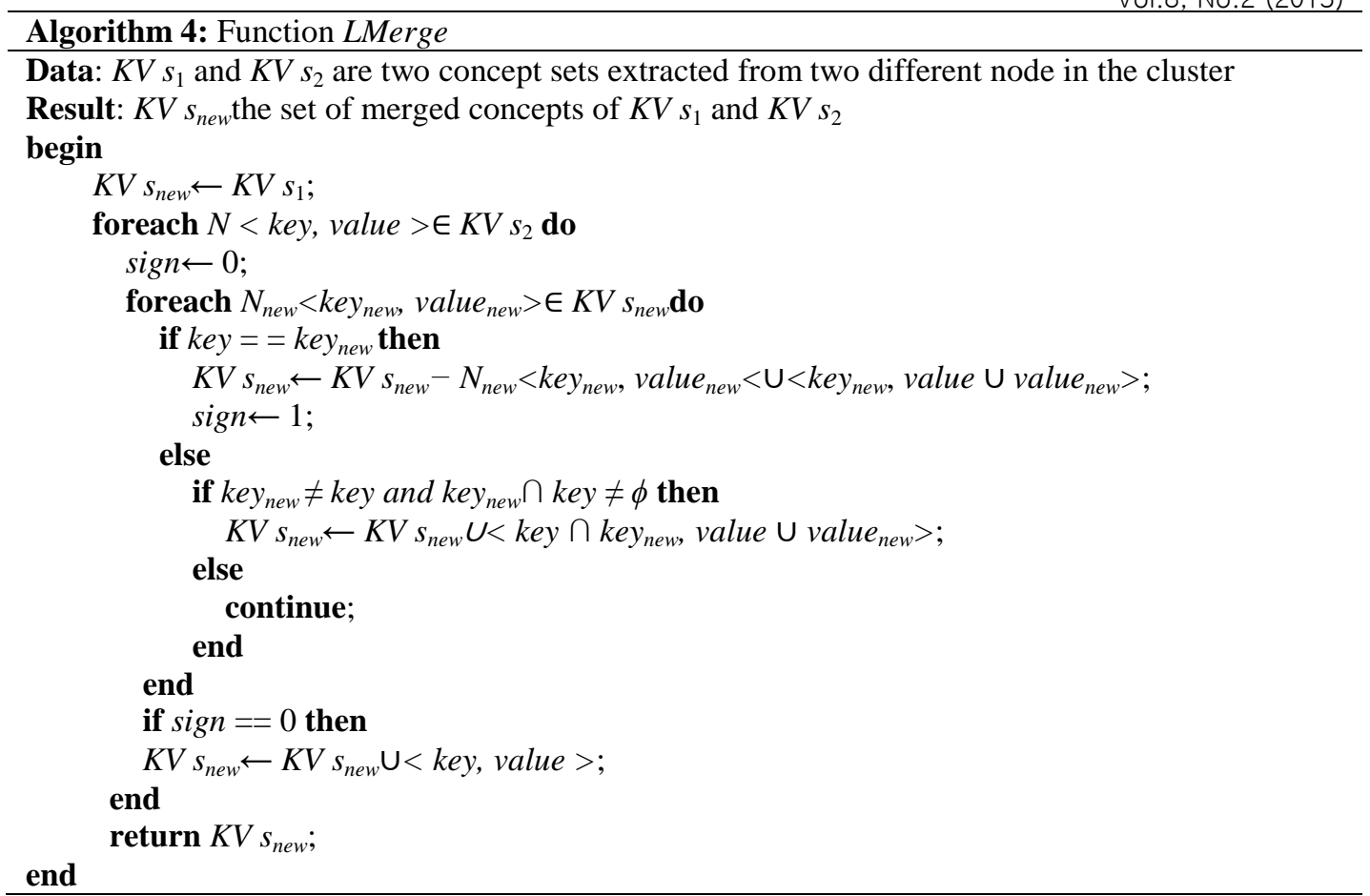

$R 1$ : If $k e y_{1}=k e y_{2}$, then the new concept will be $N_{\text {merge }(1,2)}<k e y_{1}$, value $_{1} \cup$ value $_{2}>$;

$R 2$ : If $k e y_{1} \neq k e y_{2}$ and $k e y_{1} \cap k e y_{2} \neq \phi$, then the new concept will be $N_{\text {merge(1,2) }}<k e y_{1} \cap$ $\mathrm{key}_{2}$, value $_{1} \cup$ value $_{2}>$;

$R 3$ : If $k e y_{1} \cap k e y_{2}=\phi$, then no new concept will be generated.

For each concept in the two sets of concepts being merged, in the result set, there must exist a concept with the same key. Therefore, if $R_{1}$ is not satisfied, the concept itself should be added into the result directly. This is reflected in the merge function, LMerge(see Algorithm 4).

Table 2. Structure of a Node in L-INDEX

\begin{tabular}{|l|l|l|}
\hline field & data type & description \\
\hline id & integer & the identification of the node \\
key & array & the key(terms) of the node \\
value & array & the value(documents) of the node \\
$P_{a}$ & array & the parent nodes of the node \\
$C_{h}$ & array & the child nodes of the node \\
Level & integer & the level of the node in L-INDEX \\
\hline
\end{tabular}

\section{Establishing, Maintaining and Searching on L-INDEX}

\subsection{Establishment of L-INDEX}

After computing with map function and reduce function, we get the all the concepts extracted from the documents in the corpus. The next job is to develop an approach of establishing L-INDEX that is consistent with the obtained concepts as well as the strategies of storing the structure. Definition2 defines the properties an L-INDEX. The properties also specify the relationships among the nodes in the structure. Following the definition, we develop a strategy to store the structure of $L-I N D E X$ in HBASE, a distributed, column-oriented database system widely used to manage data in MapReduce environment.

To identify the concepts, each concept is assigned an id to differential it with others. For the key and value of formal concepts are both sets, it's efficient to use array to store 
them during computing. We use $P_{a}$ and $C_{h}$ to represent the parent nodes and child node for a given concept. Obviously, both $P_{a}$ and $C_{h}$ are arrays. Level denotes the level at which the current concept is. Specially, the $i d$ of concept $\{T, \phi\}$ is set to 0 , and that of $\{\phi, D\}$ is set to -1.The full structure describing a node in $L-I N D E X$ is listed in Table 2.

Algorithm 5 expresses the algorithm used to generating the structure from the concepts extracted from a corpus. As we know, each concept in the returned set from reduce function corresponds to a node in L-INDEX. However, reduce function is not responsible for finding the relationships among concepts. Concretely, $P_{a}, C_{h}$ and Level of a node could not be set by reduce function. Actually in our work, they are computed with Algorithm 5. To make the algorithm more efficient, the concepts are sorted with the number of the documents contained in their values with function SortByNumberOfValues, and the intermediate nodes are sort by their levels in the process of computing. After executing the algorithm, each concept becomes a node with the structure defined in Table 2 which can also use to draw the relationship among nodes in $L$-INDEX. The structure can be easily managed with HBASE in MapReduce.

So far, a lattice-based index $L-I N D E X$ has been established for a corpus of documents. We will discuss how to update the structure as the documents vary in the corpus.

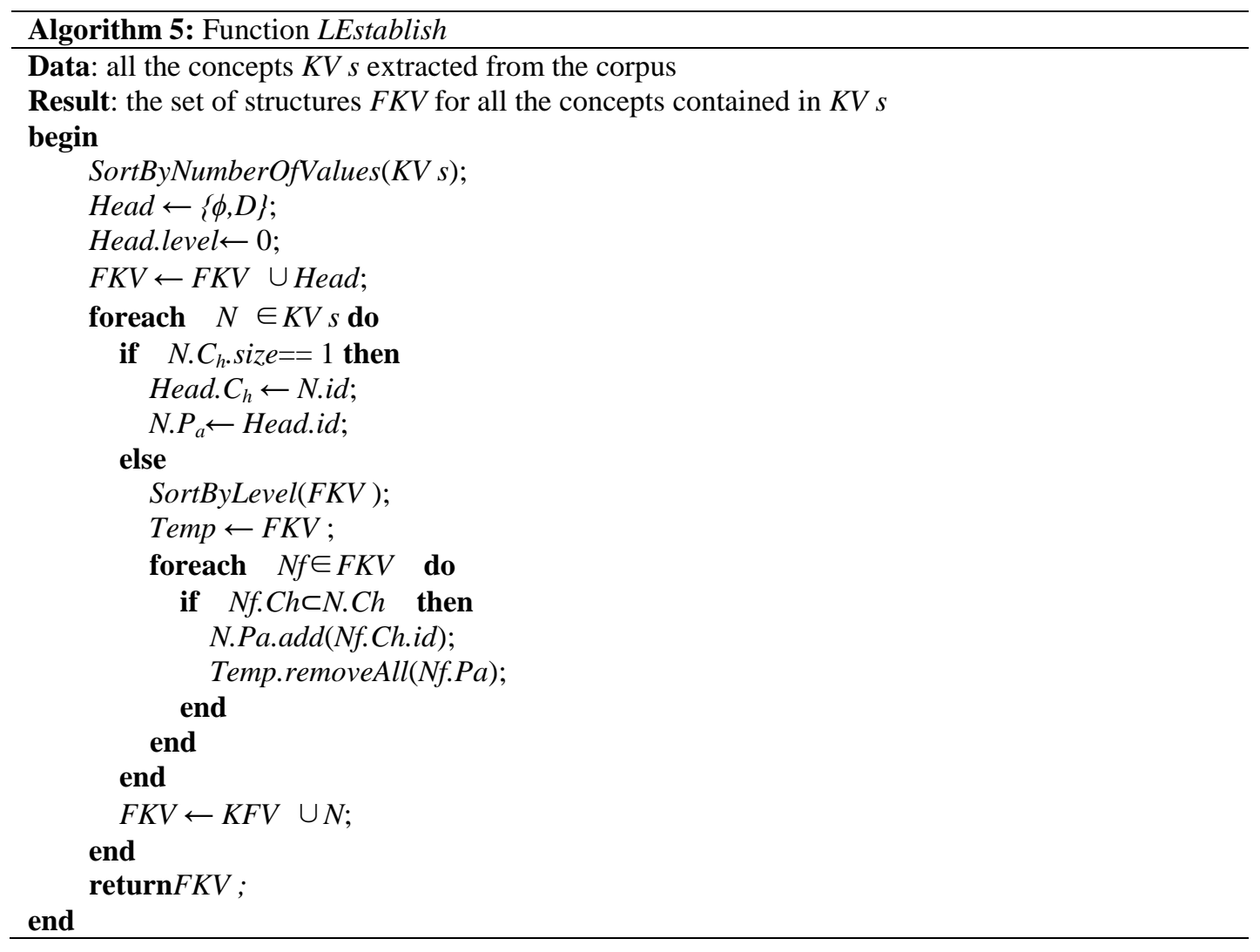

\subsection{Maintenance of L-INDEX}

In real applications, such as on the Web, the documents in the corpus always change dramatically. Easy to be maintained is very crucial for the index to be applicable in such challenging scenarios. When a document is added into or removed from the corpus, some of the index nodes need be updated. The modification can take place on their keys or values, or both of them.

The update of L-INDEX is easy when a new document is added into the corpus. The document alone can be regarded as a corpus and a special concept will be generated for it, and then revoke Function LMerge (see Algorithm 4) to update the concepts in original 
one. While, when a document $d i$ is removed from the corpus, there are three cases will happen on a given node $N(k e y, v a l u e)$ in the L-INDEX:

(i) If $\{d i\} \subset$ value, then $d i$ will be removed from the node;

(ii) If $\{d i\}=$ value, then $N$ will be removed from $L-I N D E X$;

(iii) $N$ keeps unchanged, otherwise.

Algorithm 6 implements updating when a document is removed from the corpus. It firstly calls Locate Minimal Node to locate the node $N i$ who has the minimal values containing the removed document ID. Then, it traverses the L-INDEX along the paths on which all the nodes are the ancestors of $N i$ and removes the document's ID from their extensions. Meanwhile, the system needs to check if the updated node becomes a duplicate of its child. If yes, the node will be removed from the index and corresponding modifications will also happen on the child nodes, such as changing their parent to the removed one's.

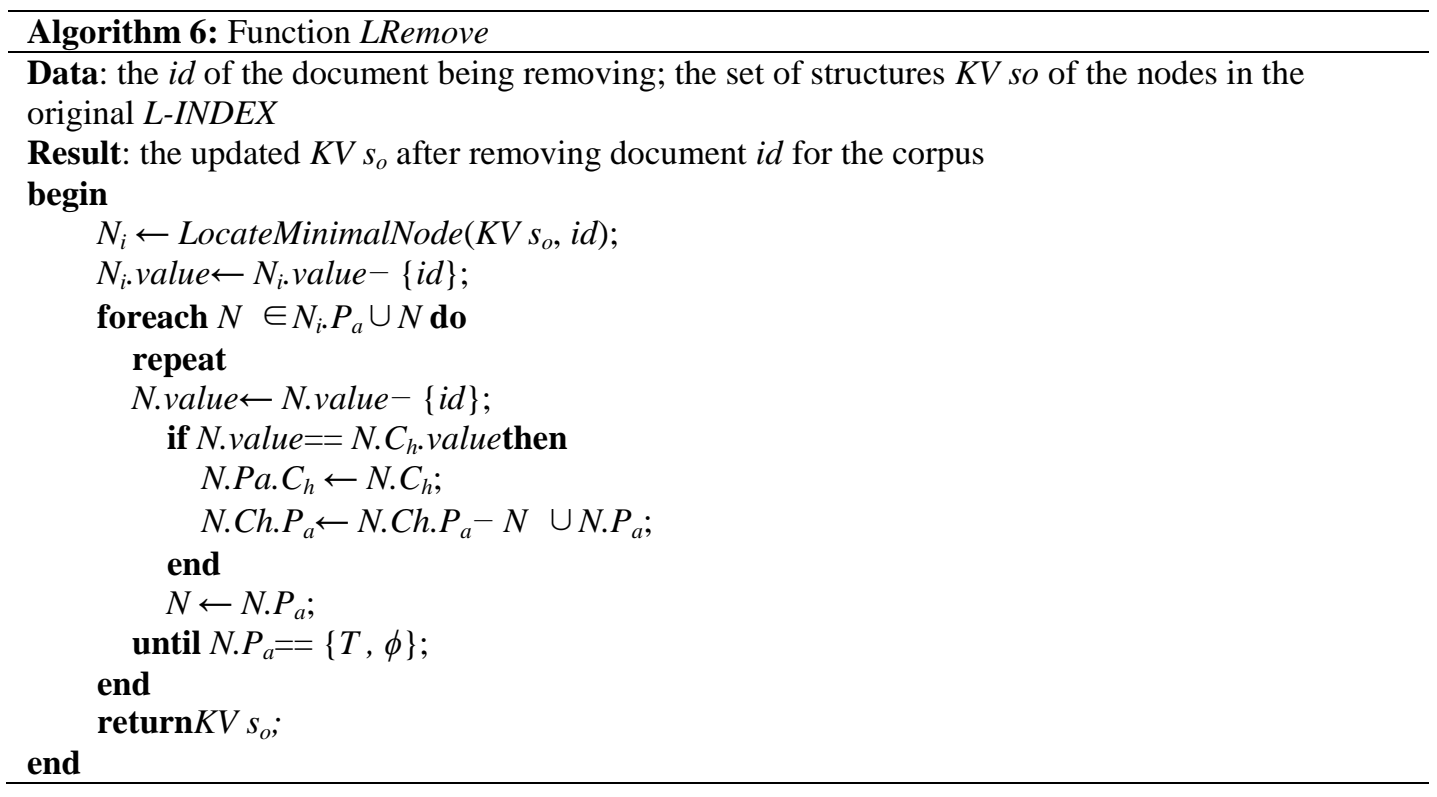

\subsection{Searching on L-INDEX}

As in our previous discussion, multiple sets of concepts are generated after executing the reduce function in the cluster. To improve performance, we build an L-INDEX for each set of concepts. This makes the individual L-INDEXes not too large and provides a good compromise for the efficiency of establishing, maintaining and searching. These relative small $L$-INDEXes help to easily employ multi-threads to speed up searching the documents in MapReduce. The number of threads depends on that of sets returned from the reduce function. In this way, a searching task is delivered to different nodes, and the results from different nodes are merged together before being responded to users.

L-INDEX supports two kinds of search: one to search the terms contained in a given document, the other is to search the documents containing same set of terms. Generally, to accomplish the first kind of search, the system traverses the index in a bottom-up way. While, for the second kind of search, the system generally takes a top-down way. For example, in Figure 1, when trying to search what terms are contained in $d o c_{2}$, we use bottom-up approach to search the index, that is, to find the lowest node whose value contains $d o c_{2}$. Node 2 is the result node, and it is easily to get all terms printer, price and car which appear in $d o c_{2}$. If we want to retrieve the documents which contain dictionary, it is efficiently to access the index in a top-down way. In other words, to seek the highest node whose key contains dictionary and the documents in its value are the results. In the figure, Node 5 is the result node and the documents in its value, $d o c_{3}, d o c_{4}$ and $d o c_{5}$, are 
returned to the user.

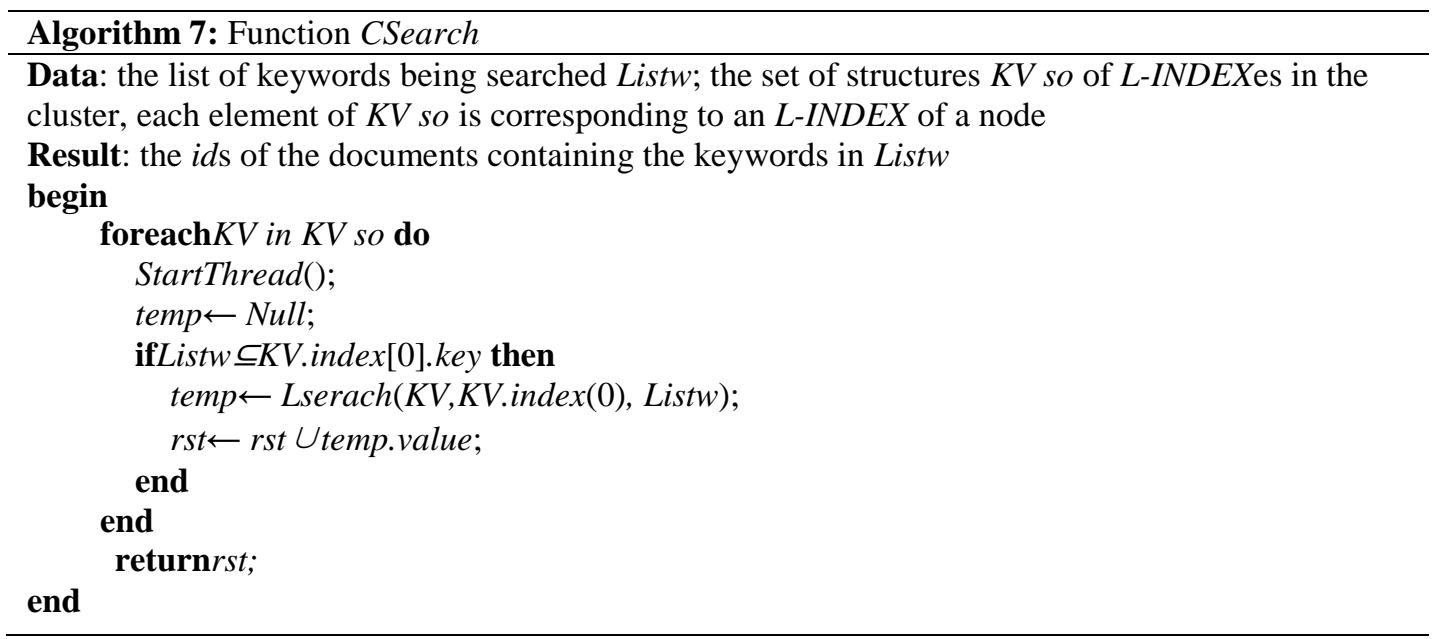

Above discussion shows there are at least three advantages of searching documents with $L-I N D E X$ in MapReduce. First, for searching multiple terms or documents, the system only needs to access the nodes with parent child relationship. Second, because $L$-INDEXes are distributed, this enables searching different nodes with multi-threads at the same. Third, as we know, if an L-INDEX is set to a proper size, it can respond the search promptly.

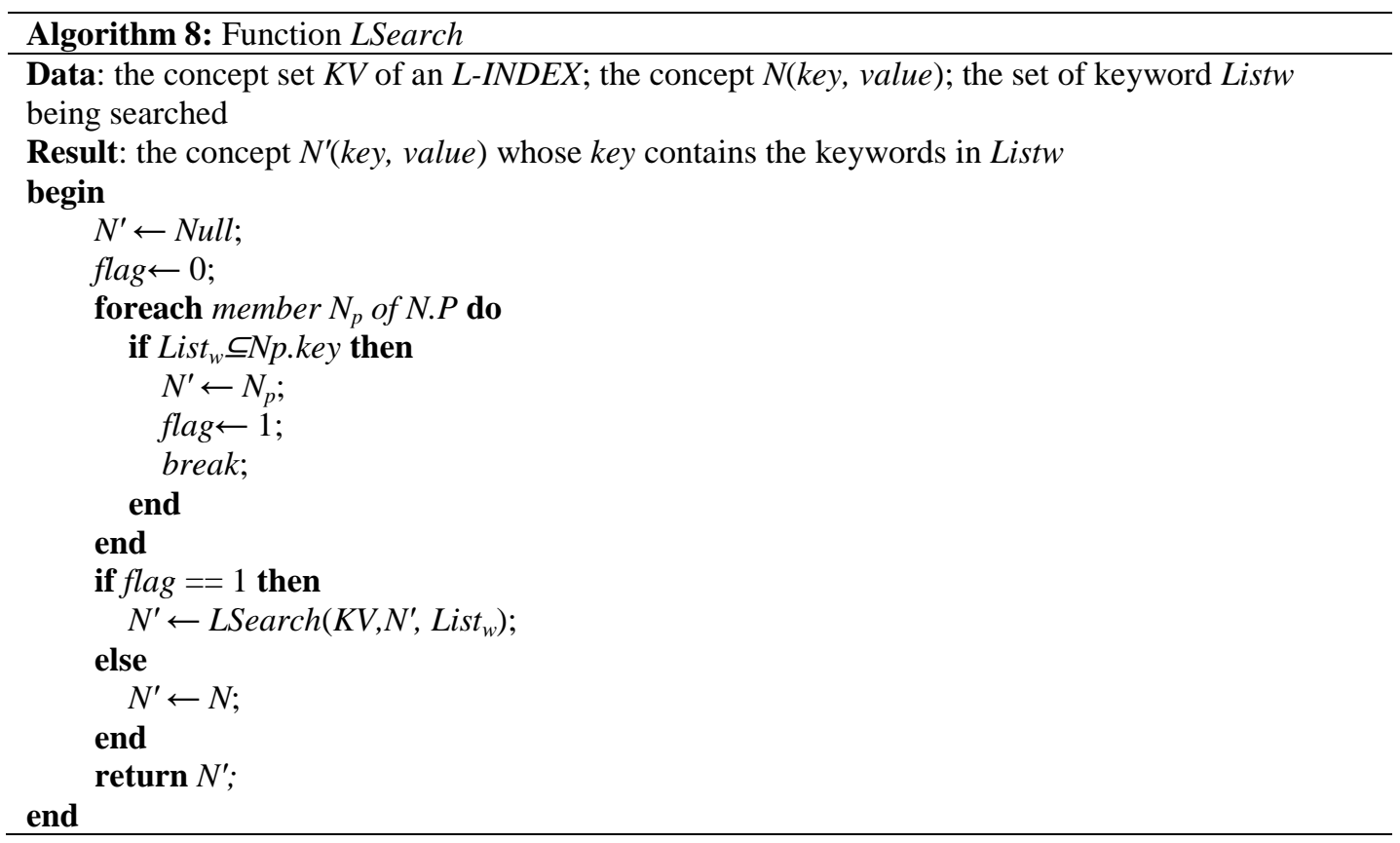

As an example, let us see how to search the documents which contain printer and dictionary in the L-INDEX shown in Figure 1. The employed strategy of searching is in top-down style, means that the search begins at the node with most values and lest keys Node $0<\phi,\left(d o c_{1}, d o c_{2}, d o c_{3}, d o c_{4}, d o c_{5}\right)>$. After arriving at Node $6<($ printer $),\left(d o c_{1}\right.$, $\left.d o c_{2}, d o c_{3}, d o c_{5}\right)>$ whose key is overlapped with \{printer, dictionary $\}$ at $\{$ printer $\}$, the algorithm will access Node $4<$ (printer, dictionary), (doc3, doc5) > which is a parent of Node 6.According to the definition of L-INDEX (see Definition 2), there does not exist any two nodes having the same key or value in the index. Therefore, the value of Node 4 is the result documents. Even there are 12 nodes in the example L-INDEX, we only need 
to access three of them by taking the advantage of their parent-child relationships during searching. This offers an illustration of the efficiency of searching documents with L-INDEX. The detailed description of the strategy is provided in Algorithm 7 and 8. The performance of the algorithms is demonstrated in Section 6.

\section{Experimental Evaluations}

In this section, we present the experimental results for the evaluation of $L$-INDEX. Three groups of experiments were conducted to measure the performance of establishment, maintenance of L-INDEX and retrieving documents on it. We start by giving an overview for our experimental settings which include the used corpora and the implementing environment which the experiments were conducted on.

\subsection{Experimental Settings}

Our experiments were carried out on the test corpus we crawled from Web. There are $5.0 \times 10^{5}$ textual articles with a raw size of about $15 \mathrm{~GB}$ and 5,000 per document in average word occurrences. After being preprocessed, the volume of articles is about $620 \mathrm{M}$ containing about 1350 different words in total.

All our codes were written in Java and performed in a 7-virtual-node cluster which was built on 7 physical machines. The machines were Inter(R) Xeon(R)CPU E3-1230 V2 @ 3.30 GHz, 4G Memory and 1T Disk using Windows 7Ultimate in 64-bit mode. The operating systems of the virtual nodes were Ubuntu 12.04. All the experiments were implemented in the environment ofHadoop-1.0.4 and HBase-0.90.4.

\subsection{Factors Affect the Performance of Establishing $L$-INDEX}

We implemented the experiments for investigating the factors affecting the performance of establishing $L-I N D E X$ from three aspects: (1) the impact of document corpus size on the time needed for computing concepts, (2) the effects of the number of sub-L-INDEXes on the efficiency of the establishment and (3) comparison of establishing $L-I N D E X$ with the existing popular distributed index structures for textual data, such as Lucene.

Effects of corpus and nodes of the cluster on the efficiency of computing concepts $L-I N D E X$ is designed for clusters, in order to make the system to have a good overall performance, the corpus on a single node should be at a proper size. Intuitively, the bigger the size of the index is, the more the time will be needed to construct it. Therefore, to examine the size of the corpus effects on the performance of establishing L-INDEX, we measured how the building time and index size vary with the tested corpus size. There are three sub-corpora on which the experiments were conducted:

- The first sub-corpus has 100,000 articles with 400 words, and is denoted as $1 \times 10^{5} \times$ 400 . The size of document context is about $120 \mathrm{M}$ bytes.

- The second sub-corpus has 200,000 articles with 800 words, and is denoted as $2 \times$ $10^{5} \times 800$. The size of the document context is about $260 \mathrm{M}$ bytes.

The third sub-corpus has 500,000 articles with 1,200 words, and is denoted as $5 \times 10^{5} \times 1$, 200 . The size of the document context is about $600 \mathrm{M}$ bytes. 


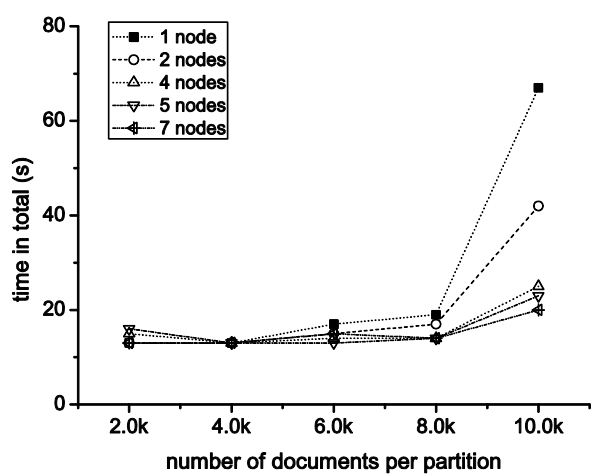

(a) Time for computing concepts $\left(1 \times 10^{5} \times 400\right)$

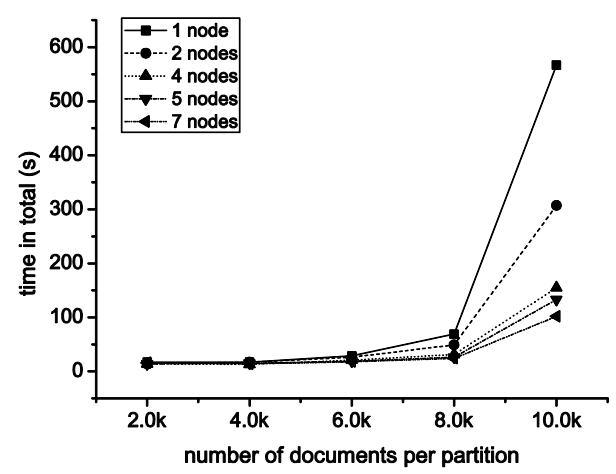

(c) Time for computing concepts $\left(2 \times 10^{5} \times 800\right)$

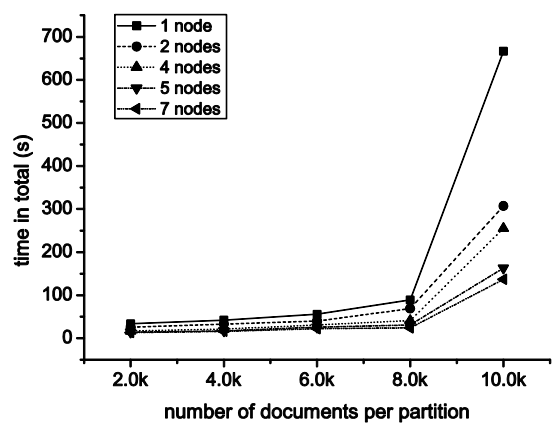

(e) Time for computing concepts $\left(5 \times 10^{5} \times 1200\right)$

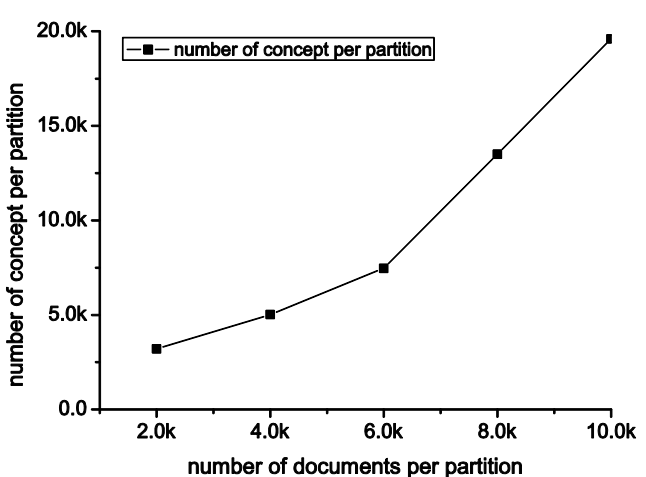

(b) Average number of concepts $\left(1 \times 10^{5} \times 400\right)$

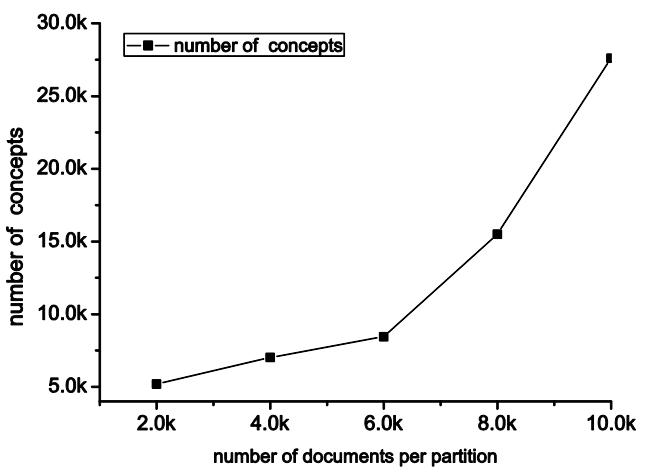

(d) Average number of concepts $\left(2 \times 10^{5} \times 800\right)$

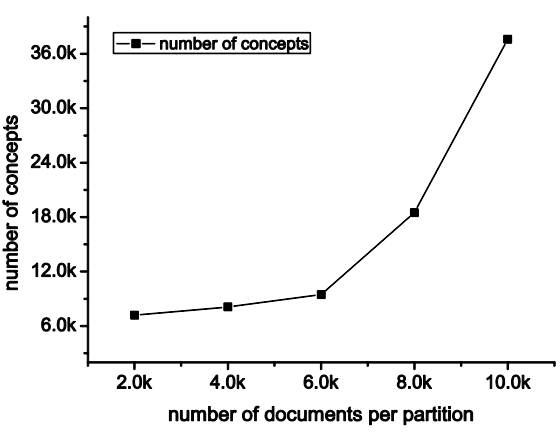

(f) Average number of concepts $\left(5 \times 10^{5} \times 1200\right)$

\section{Figure 2. Effects of the Size of Corpus and Nodes of Cluster on the Size of Document Context}

In MapReduce, all the corpora are stored on HDFS which makes it easy control the size of document context when employing LMap to generate the set of concepts. Figure 2 illustrates the effects of the size of corpora on the performance of computing concepts. Figure 2(a), 2(c) and 2(e) depict the time taken for computing the concepts varies accordingly as the size of corpus increases. These figures show as the number of nodes increases in the cluster, the overall time needed to compute the concepts decreases quickly. This feature is guaranteed by MapReduce which supports efficient distribution of 
computational tasks to different nodes. It is intuitive that as the size of corpus gets larger, the time needed to process the data becomes more. Figure 2(b), 2(d) and 2(f) display the number of concepts generated from the corresponding corpus. They give an illustration for the increment of concepts with that of the size of corpus. As the number of concepts increases, so does the time needed to generate the concepts. Therefore, on MapReduce platform, increasing the number of nodes can improve the performance of the processing.

Effects of number of sub-L-INDEXes on the performance of reduce phase as we know, when the corpus is massive, it is very time-consuming to build the index as a whole on a single node. Thus, we establish a lot of sub-L-INDEXes on different nodes instead of building an entire one on a single one. Before generating the concepts, we divide the nodes into different groups, and each group is assigned an ID. During the reduce phase, only the concepts from the nodes with the same group ID are merged together to form a sub-L-INDEX. Therefore, we need to investigate how the number of sub-L-INDEXes affects the performance of reduce phase. The time needed to execute the reduce function and the number of concepts after merging were measured for different number of sub-L-INDEXes during the experiments. The number of sub-L-INDEXes was set to 10, 20, 40, 60 and 80, respectively. The experimental results are shown in Figure 3. Figure 3(a) describes the time consumed for running reduces function, and Figure 3(b) demonstrates the average number of concepts for each sub-L-INDEX.

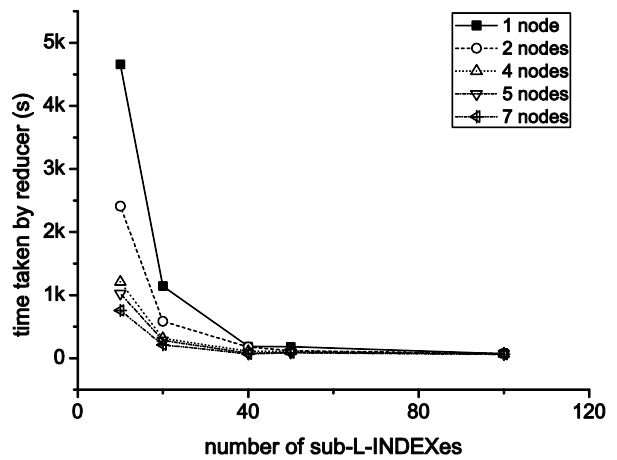

(a) Time for generating sub-LINDEXes

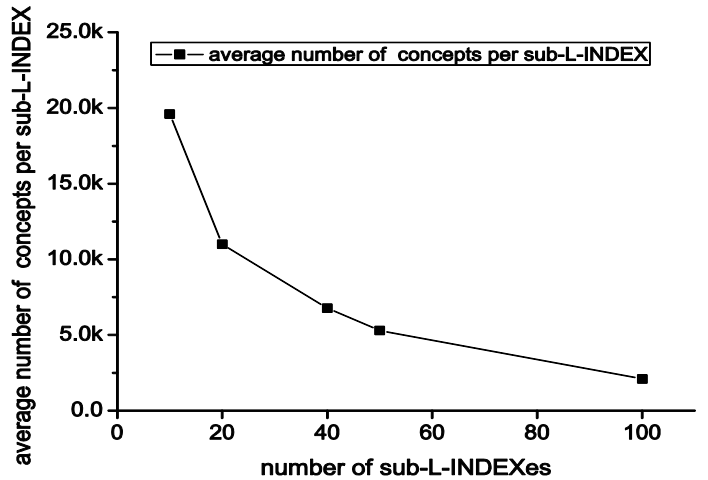

(b) Average number of concepts varies with number of sub-LINDEXes

\section{Figure 3. Impact of L-INDEX's Number on Generating Concepts}

The curve obviously indicates that the time varies inversely as the number of sub-L-INDEXes, especially when there are less than 20 sub-L-INDEXes in the cluster. During the reduce phase, with fewer sub-L-INDEXes, more concepts need be merged at a single node. While as the sub-L-INDEXes become more in the cluster, the reduce function merges fewer concepts. Likewise, when performance is better if more nodes are running reduce function. However, as the number of sub-L-INDEXes increases, the number of nodes affects the performance becomes less. This means overly few sub- L-INDEXes in the cluster will make the nodes run reduce function overloaded. When the number of sub$L-I N D E X$ es is beyond 40, increasing nodes has less impact on the overall performance of the cluster. This is caused by the increasing latency because of the increasing network traffic.

These experiments show that the indexing time increases with both the increment of corpus size and the decrement of the number of sub-L-INDEXes. Therefore, in order to improve the entire indexing performance, we need to reach a compromise between the two factors.

Comparison of the performance of building L-INDEX with that of other index's. To 
illustrate the advantage Figure 4 demonstrates the indexing time of $L$-INDEX compared with Lucene. Lucene, which was developed by Aparch.org, provides a distributed way of indexing and searching documents, and has been widely used in IR area. The experiment was conducted with 4 collections of documents. It testifies that when indexing small size of corpus, such as $(D=5 \mathrm{k}, N=7)$ and $(D=10 \mathrm{k}, N=7)$, where $D$ is number of documents for each iterator and $N$ is the nodes in the cluster, $L-I N D E X$ shows no advantage over Lucene, even Lucene performs better than L-INDEX. With the increase of corpus, L-INDEX evidently outperforms Lucene. As shown in the figure, when $\mathrm{D}=10, \mathrm{~N}=7$, the time consumed by L-INDEX is only two-third of that taken by Lucene's. This implies that increasing number of nodes will greatly improve the performance of indexing the massive corpus with L-INDEX.

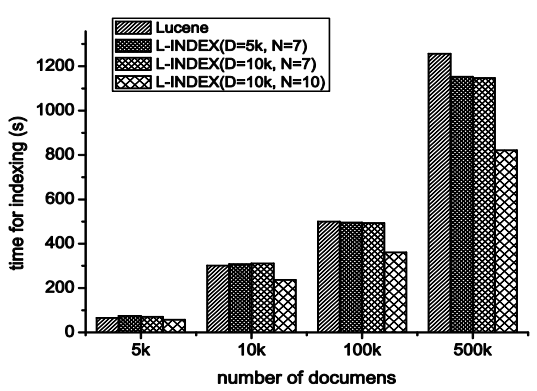

Figure 4. Comparison of Indexing Time between L-INDEX and Lucence

\subsection{Performance of Maintaining L-INDEX}

As we know, the web pages vary very rapid on the Web. In order to investigate if $L-I N D E X$ can adapt to this situation, we carried out two experiments to measure the cost of $L$-INDEX's maintenance, exactly, adding new documents and removing the existing ones for the corpus. During the experiments, we pick $D=10$ for adding new documents into $L-I N D E X$. Still, we compared the time taken for updating $L-I N D E X$ with that for the same operation in Lucene when adding new documents or removing existing ones from the corpus. The scales of corpus for changing were set to $1 \%, 5 \%$ and $10 \%$.

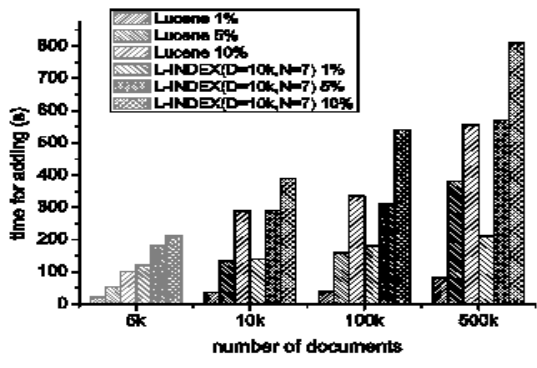

(a) Time for adding new documents

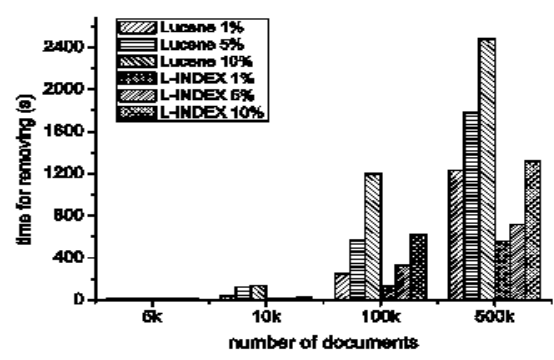

(b) Time for removing documents

Figure 5. Performance of Updating L-INDEX

Figure 5 gives description of the experimental results. Figure 5(a) illustrates that when adding new documents, Lucene shows better performance than L-INDEX. In L-INDEX, when new documents are added into the corpus, some new sub-L-INDEXes need be established before using map and reduce functions to modify the index. Therefore, the performance of updating $L$-INDEX equals to that of building a new index which depends on the number of nodes attending the computation. But for Lucene, it only updates the index itself and need not do any extra computing. This makes Lucene superior to 
L-INDEX on increment. When removing documents, like Lucene, L-INDEXneed not access the sub- $L-I N D E X$, what it needs to do is only to remove the doc-IDs of the deleted documents from the node internals which contain the documents with Algorithm 6. In contrast to adding documents, L-INDEXoutperforms Lucene for removing documents. As we know, prior to removing the document IDs from the index, first it needs to locate them in the index through query. As shown in our next experiment, the query performance of using $L-I N D E X$ is obviously better than Lucene's. It is the reason why L-INDEX takes less time for removing documents compared with Lucene.

\subsection{Retrieving on L-INDEX}

Effectiveness In information retrieval area, it is commonly to use precision and recall [13] as measures to assess the quality of the retrieved results. The two metric are defined as:

$$
\text { precision }=\frac{\text { correct } \cap \text { returned }}{\text { returned }} \quad \text { recall }=\frac{\text { correct } \cap \text { returned }}{\text { correct }}
$$

where correct is the set of correct documents in corpus which contain the searched items, and returned refers to set of documents in the retrieved results. According to the properties defined in Definition 2, there must exist a path among the documents which contains a certain set of common terms. By using the sub-sup relationship, it is easily to get all the documents containing the same set of terms. Meanwhile, searching along the path on which the node intensions contain the being searched items ensures the returned documents are correct to the search task. These observations indicate that the precision and recall of searching through L-INDEX does not depend on L-INDEX itself, but on the processing of the documents before building the index. Therefore, instead of implementing some experiments to demonstrate the effectiveness of searching documents through $L$-INDEX, we demonstrate its performance with following experiment.

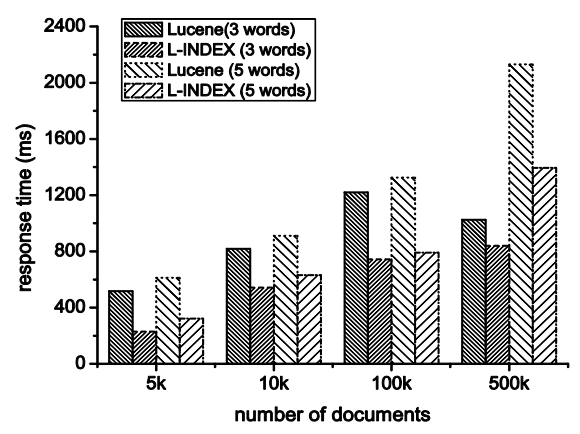

Figure 6. Query Performance

Performance This experiment tries to investigate the performance of querying documents containing certain keywords through L-INDEX. We measured the response time for query documents containing different sets of words. Figure 6 depicts the average response time for querying 3 or 5 words through L-INDEX and Lucene. In which, the average time was obtained through executing 100 queries of words randomly picked from the dictionary. Although when querying on $L-I N D E X$, it needs to merge the intermediate results returned from different nodes, L-INDEX still could respond queries more efficiently than Lucene. That is because as we discuss in Section 5.3, when querying on $L$-INDEX in MapReduce, multiple threads are applied to run the query in a distributed and parallel way, and in addition, on each sub-L-INDEX the search space is pruned significantly by exploiting the relationships between the concepts. 


\section{Conclusion}

Indexing plays a critical role for enhancing the performance of information retrieval systems, especially when the corpus contains massive documents. Developing an efficient indexing structure for massive documents becomes a hotspot of research in recent years. MapReduce provides a platform for building distributed index of textual data in a convenient way. In this paper, we address the problem of indexing massive documents in MapReduce. Different from other existing MapReduce based distributed indexing structures, we propose a novel conceptual structure for indexing massive textual data, which is called as $L-I N D E X$ and based on formal concept lattice.

With $L$-INDEX, the documents are organized together in a concept lattice which takes advantage of the relationship among documents. This feature guarantees that searching on $L-I N D E X$ with high precision and recall grain. Besides, the algorithms for establishing, maintaining $L-I N D E X$ and searching on $L-I N D E X$ on MapReduce platform have been presented in this paper. The performance of the proposed index has been verified with experiments from various aspects. The results of the experiments demonstrate that $L$-INDEX has good performance for retrieving documents compared with some existing ones, such as that used in Lucene. However, $L-I N D E X$ still has some disadvantages, such as does not perform well when updating for new documents added into the corpus. Therefore, in our future work, we will try to develop some effective strategies to improve the efficiency for increasing the structure.

\section{Acknowledgements}

The authors gratefully acknowledge the support of Shanghai Key Science and Technology Project in Information Technology Field (14511107902), Shanghai Leading Academic Discipline Project (No. XTKX2012) and Shanghai Engineering Research Center Project (GCZX14014, 14001).

\section{References}

[1] B. Ganter and R. Wille, "Formal Concept Analysis: Mathematical Foundations", Heidelberg, Ger. Springer Verlag, (1999), pp. 21-83.

[2] D. Jeffrey and G. Sanjay, "MapReduce: Simplified Data Processing on Large Clusters", In Proceedings of the 6th Symposium on Operating System Design \& Implementation, (2004), pp. 137-150.

[3] B. Dhruba, "The Hadoop Distributed File System: Architecture and Design", (2007).

[4] B. A. Ribeiro-Neto, E. S. de Moura, M. S. Neubert and N. Ziviani, "Efficient Distributed Algorithms to Build Inverted Files", In Proceedings of the 22nd Annual International ACM SIGIR Conference on Research and Development in Information Retrieval, (1999), pp. 105-112.

[5] S. Melnik, S. Raghavan, B. Yang and H. Garcia-Molina, "Building a Distributed Full-text Index for the Web", In Proceedings of the 10th international conference on World Wide Web, (2001), pp. 396-406.

[6] C. D. Manning, P. Raghavan and H. Schtze, "Introduction to Information Retrieval", Cambridge University Press, (2008).

[7] M. Cafarella and D. Cutting, "Building Nutch: Open Source Search", Queue, vol. 2, no. 2, (2004), pp. 54-61.

[8] J. Lin, D. Metzler, T. Elsayed and L. Wang, "Of Ivory and Smurfs: LoxodontanMapReduce Experiments for Web Search," In Proceedings of the 18th Text Tetrieval Conference, (2009), pp. 125-134.

[9] R. M. C. McCreadie, C. Macdonald and I. Ounis, "On Single-pass Indexing with MapReduce", In Proceedings of the 32nd international ACM SIGIR conference on Research and Development in Information Retrieval, (2009), pp. 742-743.

[10] C. A. Kumar, "Designing Role-based Access Control Using Formal Concept Analysis", Security and Communication Networks (SCN), vol. 6, no. 3, (2013), pp. 373-383.

[11] B. K. Tripathy, D. P. Acharjya and V. Cynthya, "A Framework for Intelligent Medical Diagnosis using Rough Set with Formal Concept Analysis CoRR", abs/1301.6011, (2013).

[12] I. H. Witten, A. Moffat and T. C. Bell, "Managing gigabytes: Compressing and Indexing Documents and Images", Morgan Kaufman, (1999).

[13] R. Baeza-Yates and B. Ribeiro-Neto, "Modern Information Retrieval: Retrieval: The Concepts and Technology behind Search", ACM Press, (2011). 
[14] M. Donald, N. Jasmine, C. Hang and R. Srihari, "Building Enriched Document Representations using Aggregated Anchor Text", In Proceedings of the 32nd Annual International ACM SIGIR Conference on Research and Development in Information Retrieval (SIGIR 2009), (2009), pp. 219-226.

[15] V. Anh and A. Moffat, "Inverted Index Compression using Word-aligned Binary Codes", Inf. Retrieval, vol. 8, no. 1, (2005). pp. 151-166.

[16] Y. Hao, D. Shuai and S. Torsten, "Inverted Index Compression and Query Processing with Optimized Document Ordering", In Proceedings of 18th International World Wide Web Conference (WWW2009), (2009), pp. 401-410.

[17] D. M. Christopher, R. Prabhakar and S. Hinrich, "Introduction to Information Retrieval", Cambridge University Press, (2008).

[18] M. Sergey, R. Sriram, Y. Beverly and H. Garcia-Molina, "Building a Distributed Full-Text Index for the Web", In Proceedings of 19th International World Wide Web Conference (WWW2001), (2001), pp. 396-406.

[19] A. Ahmad, T. Mohammad and P. O. Michael, "An Investigation into Improving the Load Balance for Term-Based Partitioning", Lecture Notes in Business Information Processing, vol. 5, (2008), pp. 380-392.

[20] J. Zobel and A. Moffat, "Inverted Files for Text Search Engines", ACM Computing Surveys (CSUR), vol. 38, no. 2, (2006).

[21] M. Richard, M. Craig and O. Iadh, "MapReduce Indexing Strategies: Studying Scalability and Efficiency", Information Processing and Management, doi:10.1016/j.ipm.2010.12.003, (2011).

[22] P. Denys, G. Malcom and M. Andrian, "Concept Location using Formal Concept Analysis and Information Retrieval', ACM Trans. Softw.Eng. Methodol. (TOSEM), vol. 21, no. 4, (2012), pp. 23.

[23] B. Ganter, "Two Basic Algorithms in Concept Analysis", FB4-Preprint, TH Darmstadt, no. 831, (1984).

\section{Authors}

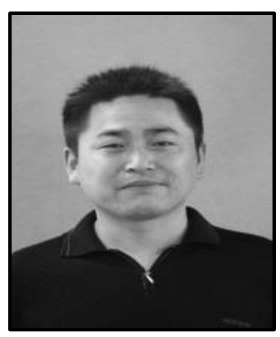

Dunlu Peng, is a professor of University of Shanghai for Science and Technology, Shanghai, China. He received his Ph.D degree from Fudan University, Shanghai, China in June 2006. He served as a PC member of SCC, EDOC, APSCC, WHICEB, CLOUD, etc. His research interests include Cloud Computing, Web applications, service-oriented computing, XML data management and Web mining.

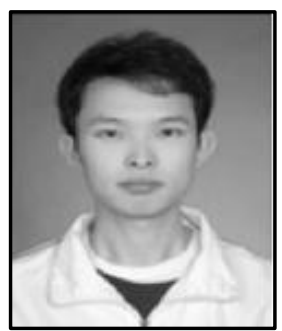

Kai Duan, is a master degree candidate of University of Shanghai for Science and Technology, Shanghai, China. He received his bachelor's degree from University of Shanghai for Science and Technology, Shanghai, China in June 2011. His research interests include Cloud Computing, Service-oriented computing and Web mining.

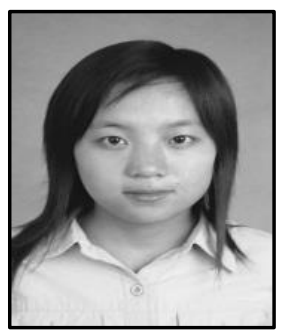

Lei Xie, is a master degree candidate of University of Shanghai for Science and Technology, Shanghai, China. She received her bachelor's degree from University of Shanghai for Science and Technology, Shanghai, China in June 2011. Her research interests include Cloud Computing, Web Mashup application and Web mining. 
International Journal of Grid Distribution Computing

Vol.8, No.2 (2015) 